\title{
Analysis of Preprocessing Algorithms for Space Frequency and Mathematical Morphology on Mammograms
}

\author{
Ramón 0. Guardado-Medina1, Luis E. Mendoza-Luna², Jorge A. Ortiz-Ramirez², \\ Humberto Bracamontes del Toro ${ }^{2}$, Ruben Ruelas Lepe ${ }^{1,2}$, Gustavo Ochoa Mata $^{3}$ \\ ${ }^{1}$ Information Technologies Department, University of Guadalajara, México \\ ${ }^{2}$ Electronics Engineering Department, Technological Institute of Ciudad Guzmán, México \\ Email: osvaldo.guardado@cucea.udg.mx
}

Received 4 August 2014; revised 26 September 2014; accepted 27 October 2014

Copyright (C) 2014 by authors and OALib.

This work is licensed under the Creative Commons Attribution International License (CC BY).

http://creativecommons.org/licenses/by/4.0/

(c) (i) Open Access

\begin{abstract}
This paper presents the performance analysis of preprocessing algorithms for enhancement features on mammograms with the objective of improving future clustering processing investigations, space frequency filtering and morphologic space filtering, which are analyzed with their different techniques in regions of interest using the DDSM database. Displaying microcalcifications, which is achieved with the Gaussian high-pass filter in the space frequency and diamond filter, in morphologic space, supported by spectral images (spectrograms) as well as the efficiency, is measured over massive preprocessing images. However, when an improved visualization is presented in processing times, it is observed that the variance in time analyzes a large number of images. Also, although the frequency is faster than morphological space, the morphological filters (Ball filtering) shows a significant result than frequency filters.
\end{abstract}

\section{Keywords}

Preprocessing Images, Mathematical Morphology, Fourier Transform, Frequency

Subject Areas: Oncology, Breast Cancer

\section{Introduction}

Breast cancer has a high level of death rate. According to estimations, in 2012, 1,151,298 new cases of breast cancer were diagnosed; 404,712 deaths were reported; and over 4.4 millions of women lived with this disease around the globe [1] [2]. In Mexico, according to Instituto Nacional de Estadística y Geografía (INEGI), on the release No. 267/11 issued on June 13, 2011, on the year of 2009, the death rate by breast cancer on the 10 states 
with highest levels of diagnosed cases is 14.4 deaths for every 100 thousand women over 25 years old, which is higher than the double average on the 10 states with minor diagnosed cases (7.2 death women for every 100 thousand over 25 years old). Image diagnostic is a tool that helps present medicine on breast cancer detection. Magnetic Resonance Images (MRI), Computed Tomography (CT), Digital Mammography (DM), among other techniques are very effective non-invasive ways that allow to analyze inner anatomy in the search of abnormalities [3] [4]. These techniques have increased on the medical investigation field, providing alternatives on the detection of the disease, or even preventing the beginning of the illness. Therefore, images are a very effective way of detection and location of abnormalities, helping on the diagnosis. In the image processing field, features enhancement, intensity manipulation, noise reduction, removal of background, are an important part of the preprocessing stage. The last one has the purpose of enhancing the image appearance, enhancing the microcalcifications features separating breast tissue. A lot of tools are available for their use, being the most well-known: frequency, morphological and multispectral filtering, and using a specific filter depends on the type of the image and the results desired. In this investigation extraction filters and enhancement features were selected in frequency domain and mathematical morphology. According to [5], the geometrical structure of the image is determined by a local comparison and the predefined elements called: structural elements (SE). Processing an image using morphology is based on the relation of the two sets: the image itself and the structural element, which usually is smaller than the image. Depending on the shape and size of the structural element, different results can be achieved.

Some studies have made the detection of microcalcifications [6] [7]. It explains the preprocessing done with tophat (mathematical morphology), which is important to mention that this step facilitates the application of techniques of segmentation or neural networks. On the other hand, some other studies did not take into consideration this situation for example in [8]-[10]. Therefore, this study shows the behavior of the main algorithms, which provides important information in subsequent processing.

\subsection{Power-Law Transformations and Frequency Space}

The $T$ transformation is the neighborhood size $1 \times 1$ (one pixel). In this case, $g$ value on $(x, y)$ depends only on the intensity of $f$ in that point, and $T$ becomes an intensity transformation function or gray-level function. As they depend only on intensity values, and not explicitly on $(x, y)$, these functions are written as

$$
s=T(r)
$$

where $r$ denotes the intensity of $f$ and $s$ the intensity of $g$, both on its only point $(x, y)$ on the image. As seen on Figure 1. This function transforms the intensity values of the image $f$ to new $g$ values. The gamma parameter specifies the form of the curve $y$, in case of omission, it takes the value 1 .

The shown function on the next Figure 2 is called contrast narrowing transformation, because it compresses the lower input levels less than $m$ to a narrower degree of dark levels on the output image. Also, it compresses the values higher than $m$ to a narrower band of white levels on the output. The result is a higher contrast image.

The function represented on this graphic has the form

$$
s=T(r)=1 / 1(1+(m / r) E)
$$

where $r$ represents the intensities of the input image, $s$ represents the intensity values on the output image, and $E$ controls the pendant of the function. If filters are used on frequency domain, follow these steps (also shown in Figure 3):

1) Multiply each input $f(x, y)$ by $(-1)^{x+y}$.

2) Transform the image to frequency domain by using the Fourier Discret Transformed, $F(u, v)$.

3) Multiply by a frequency filter $H(u, v)$, for each $(u, v): G(u, v)=H(u, v) \quad F(u, v)$.

4) Inverse the FDT of $G(u, v)$ (real part), retourning to space domain.

5) Multiply again by $(-1)^{x+y}[12][13]$.

\subsection{Morphology}

The word morphology commonly denotes a branch of biology that deals with the form and structure of animals 

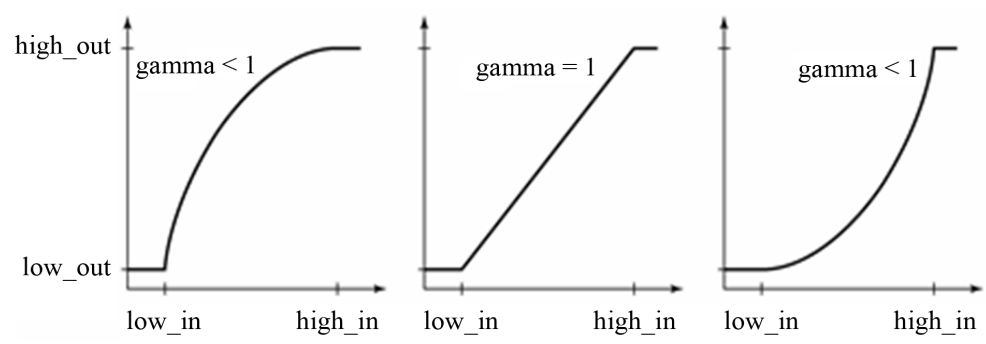

Figure 1. Mapping gamma values $g$ [11].
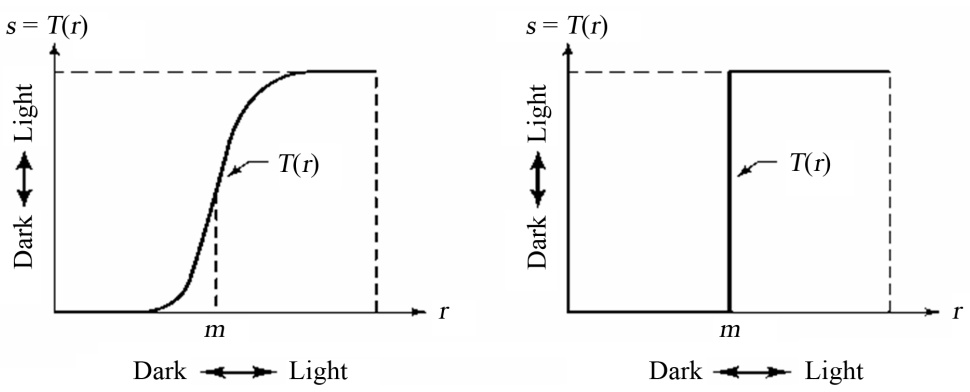

Figure 2. Contrast transformation [11].

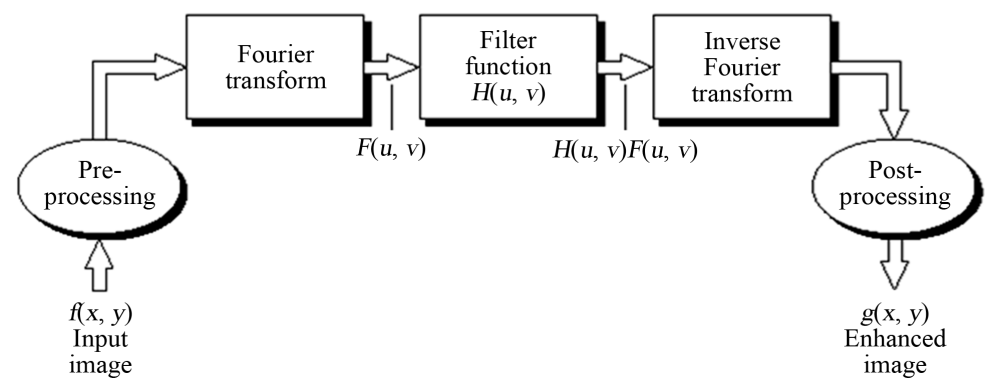

Figure 3. Block diagram of frequency images preprocessing [13].

and plants. We use the same word here in the context of mathematical morphology as a tool for extracting image components that are useful in the representation and description of region shape, such as boundaries, skeletons, and the convex hull. We are interested also in morphological techniques for pre- or post-processing, such as morphological filtering, thinning, and pruning. The language of mathematical morphology is set theory. As such, morphology offers a unified and powerful approach to numerous image processing problems. Sets in mathematical morphology represent objects in an image. For example, the set of all black pixels in a binary image is a complete morphological description of the image. In binary images, the sets in question are members of the 2-D integer space $Z^{\prime}$, where each element of a set is a tuple (2-D vector) whose coordinates are the $(x, y)$ coordinates of a black (or white, depending on convention) pixel in the image. Gray-scale digital images can be represented as sets whose components are in Z3. In this case, two components of each element of the set refer to the coordinates of a pixel, and the third corresponds to its discrete gray-level value. Sets in higher dimensional spaces can contain other image attributes, such as color and time varying components.

\subsection{Dilation}

With $A$ y $B$ as sets in $Z$, the dilation of $A$ by $B$, denoted $A \oplus B$, is defined as

$$
A \oplus B=\left\{Z \mid(\hat{B})_{z} \cap A \neq \varnothing\right\}
$$

This equation is based on obtaining the reflection of $B$ about its origin and shifting this reflection by $Z$. The dilation of $A$ by $B$ then is the set of all displacements, $Z$, such that $B$ and $A$ overlap by at least one element. 
Based on this interpretation, Equation (1) may be rewritten as

$$
A \oplus B=\left\{Z \mid\left[(\hat{B})_{Z} \cap A\right] \subseteq A\right\}
$$

Set $B$ is commonly referred to as the structuring element in dilation, as well as in other morphological operations.

\subsection{Erosion}

For sets $A$ and $B$ in $Z$ the erosion of $A$ by $B$, denoted $A \ominus B$ is defined as

$$
A \odot B=\left\{Z \mid(B)_{z} \subseteq A\right\}
$$

This equation indicates that the erosion of $A$ by $B$ is the set of all points $Z$ such that $B$, translated by $Z$, is contained in $A$. As in the case of dilation, Equation (13) is not the only definition of erosion. However, it is usually favored in practical implementations of morphology for the same reasons stated earlier in connection with Equation (5).

\subsection{Method}

To perform performance testing on preprocessing algorithms, Figure 4 shows the steps through the investigation. First, the DDSM (Digital Database for Screening Mammography) database was selected, which is a public resource for investigators. Primary support for this project was a grant from the Breast Cancer Research Program of the U.S. Army Medical Research and Materiel Command. The DDSM project is a collaborative effort involving co-p.i.s at the Massachusetts General Hospital (D. Kopans, R. Moore), the University of South Florida (K. Bowyer), and Sandia National Laboratories (P. Kegelmeyer). Additional cases from Washington University School of Medicine were provided by Peter E. Shile, MD, Assistant Professor of Radiology and Internal Medicine. Additional collaborating institutions include Wake Forest University School of Medicine (Departments of Medical Engineering and Radiology), Sacred Heart Hospital and ISMD, Incorporated. The primary purpose of the database is to facilitate sound research in the development of computer algorithms to aid in screening. Secondary purposes of the database may include the development of algorithms to aid in the diagnosis and the development of teaching or training aids [14].

This database has 2479 cases on 41 volumes (Until October 2013), which are distributed on: 695 on 12 volumes labeled as normal; 914 on 15 volumes labeled as cancer and 870 on 14 volumes classified as benign. The volumes are classified as: normal, cancer and benign; Normal cases are formed from a normal detection previous exam (taken from a file) for a patient with a normal exam, at least, four years later. A normal detection exam is where more studies are no longer needed. Cancer cases are formed from detection exams in which are found cancer pathologies. Benign cases are formed from detection exams where suspicious pathologies are found, but they are not malignant. To perform the testing 60 cases were selected from 3 different volumes (cancer_01, cancer_04, cancer_07) over 15 cancer volumes previously diagnosed (25 from cancer_01, 14 from cancer_04 and 21 from cancer_07). This is because the selected cases are those with abnormalities on microcalcifications, checked by DDSM, which were processed by two filtering methods (morphology and frequency) to find the best optimal and provide the best result to its implementation on segmentation and classification algorithms.

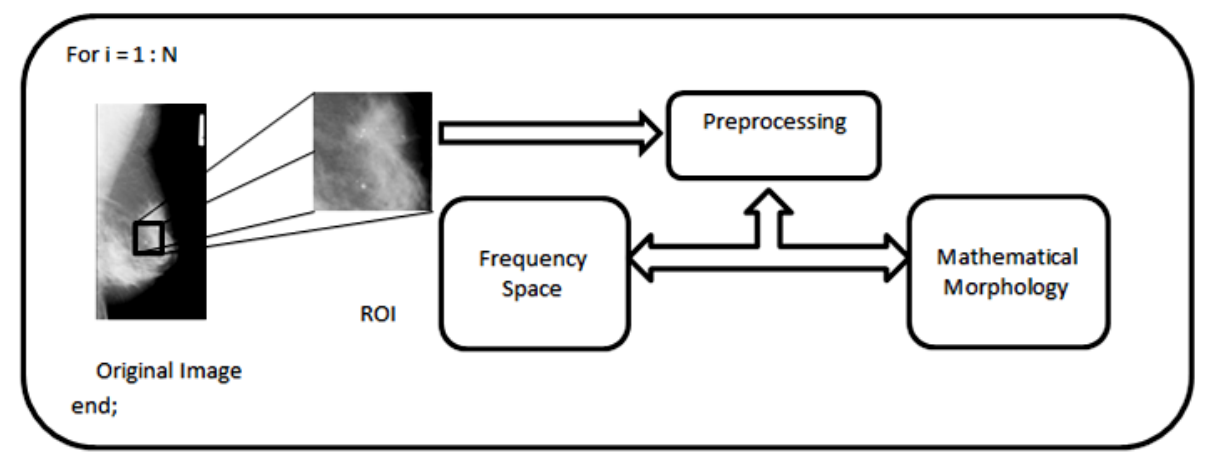

Figure 4. Performance measure methods of preprocessing algorithms. 


\section{Results}

During the algorithms application shown on Figure 4, it was observed that on frequency space, specially low-pass filter, on Gaussian and Butterworth techniques, images do not show feature enhancement, which is not helpful to the extraction of knowledge and cannot be applied on future processes, therefore, it has been discarded from the investigation.

However, high-pass filter shows a clearly visible feature enhancement on frequency space. Figure 5 shows the original images taken from DDSM database. It can be seen that the filter on Figure 6, Gaussian technique, gets rid of tissue and enhances microcalcifications; however, the filtering does not remove all the tissue from the breast, which has the same gray-level than microcalcifications. This will bring identification class problems when classification algorithms are applied. Figure 7 shows that morphology filtering, applying diamond technique, which enhances features on the image, being better than frequency space filtering, due to tissue elimination and enhancing of possible microcalcifications. Then, every stage on this investigation is detailed.

On previous Figures 8-10 it could be seen the energy distribution on every ROI on its original format, frequency space filtering and morphology space filtering, unlike a histogram, they allow to observe or to watch the original context of images that show feature enhancement, that morphology preprocessing helps to get rid of tissue from breast. This tool also helps to compare between original and processed images, so it can be seen if filtering caused unwanted alterations to the image, taking out desired features that can be diagnosed as false positives or false negatives in future processes. Finally a time test is shown on all algorithms made for frequency and morphology processing, to show how fast images can be processed on the search of microcalcifications. PC specifications are: CPU Intel i5 34503.1 GHz, 8 GB RAM 1600 MHz, HD 1 TB SATA-III, OS Windows 7 Ultimate

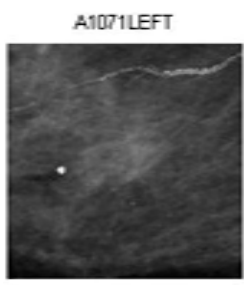

A1261LEFT

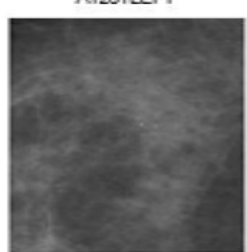

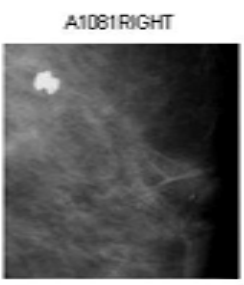

B3008PIGHT

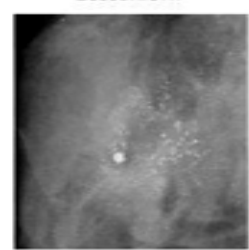

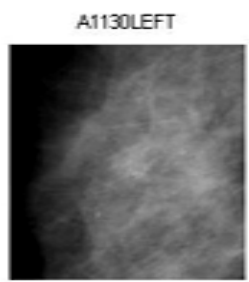

B3025PIGHT

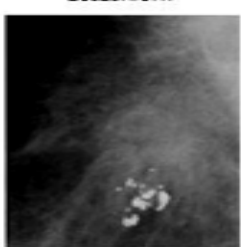

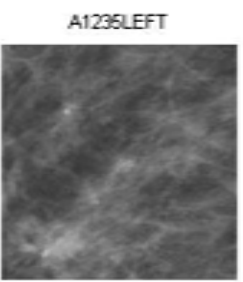

B3006PIGHT

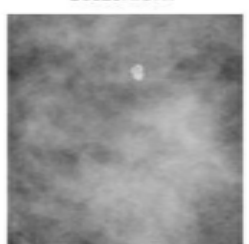

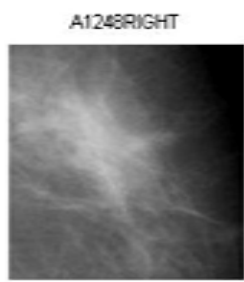

BSOSOLEFT

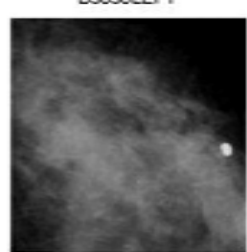

Figure 5. ROI's of the original images (A1248, A1235, A1130, A1081, A1071, B3030, B3026, B3025, B3008, A1261).

A1071LEFT FM

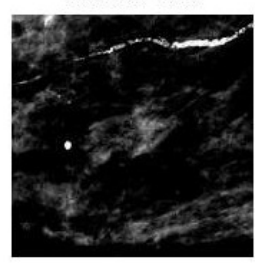

A1261 LEFT FM

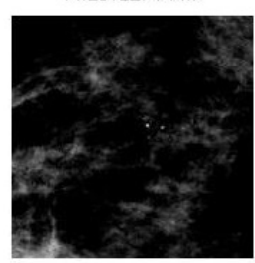

A1081RIGHT FM

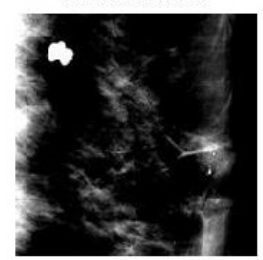

B3008RIGHT FM

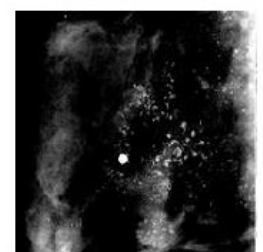

A1130LEFT FM

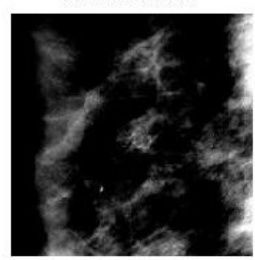

B3025RIGHT FM

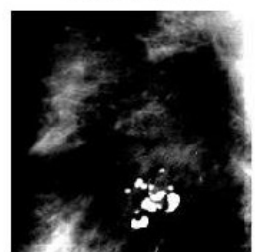

A1235LEFT FM

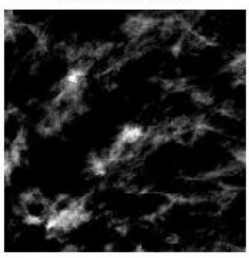

B3026RIGHT FM

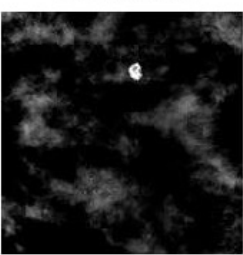

A1248RIGHT FM

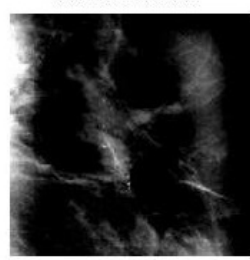

B3030LEFT FM

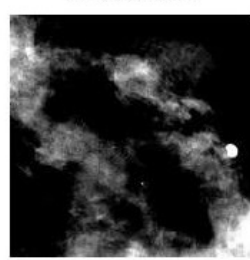

Figure 6. Feature enhancement using frequency space filtering (Gaussian filtering). 
A1071LEFT FM

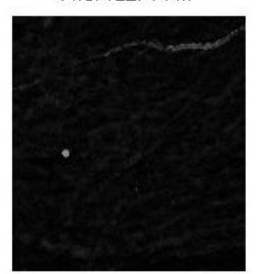

A1261LEFT FM

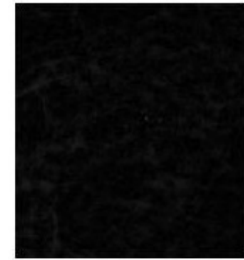

A1081RIGHT FM

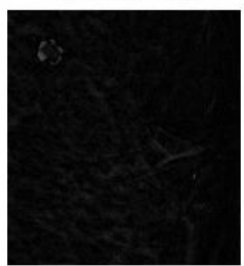

B3008RIGHT FM

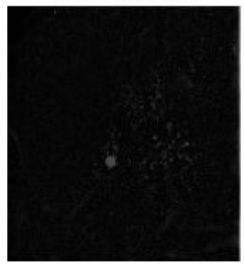

A1130LEFT FM

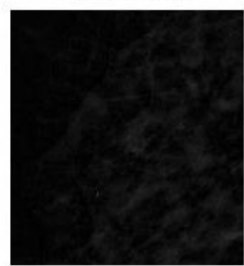

B3025RIGHT FM

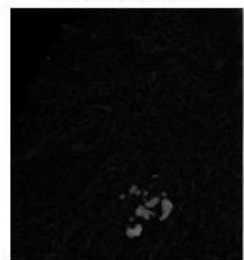

A1235LEFT FM

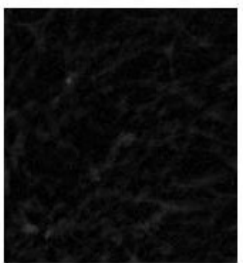

B3026RIGHT FM

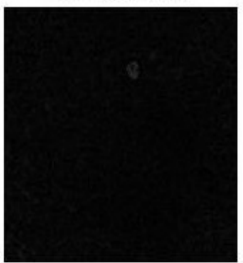

A1248RIGHT FM

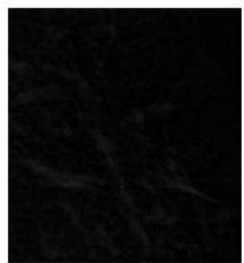

B3030LEFT FM

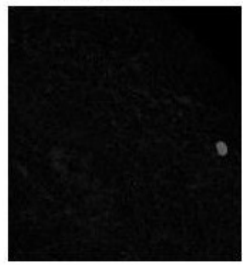

Figure 7. Feature enhancements using morphologic filtering (Ball filtering).
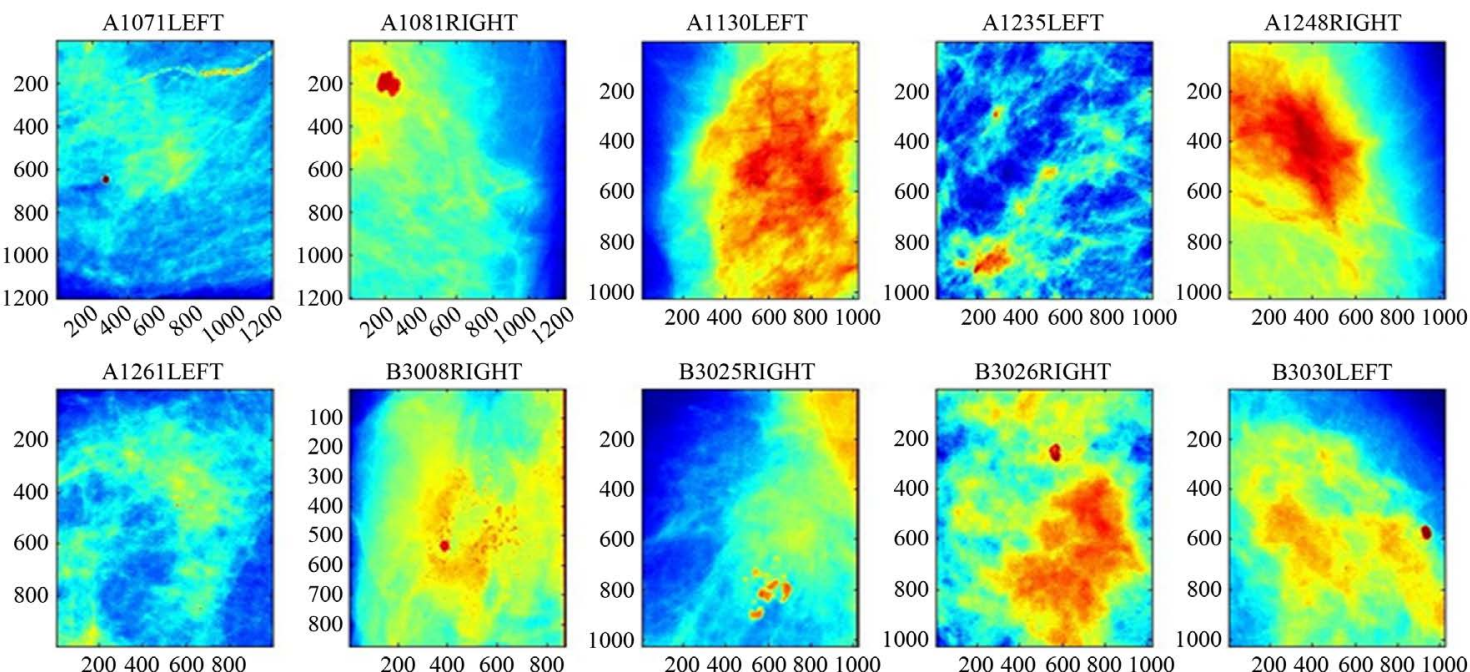

Figure 8. Original ROI’s spectrograms with size $1024 \times 1024$.
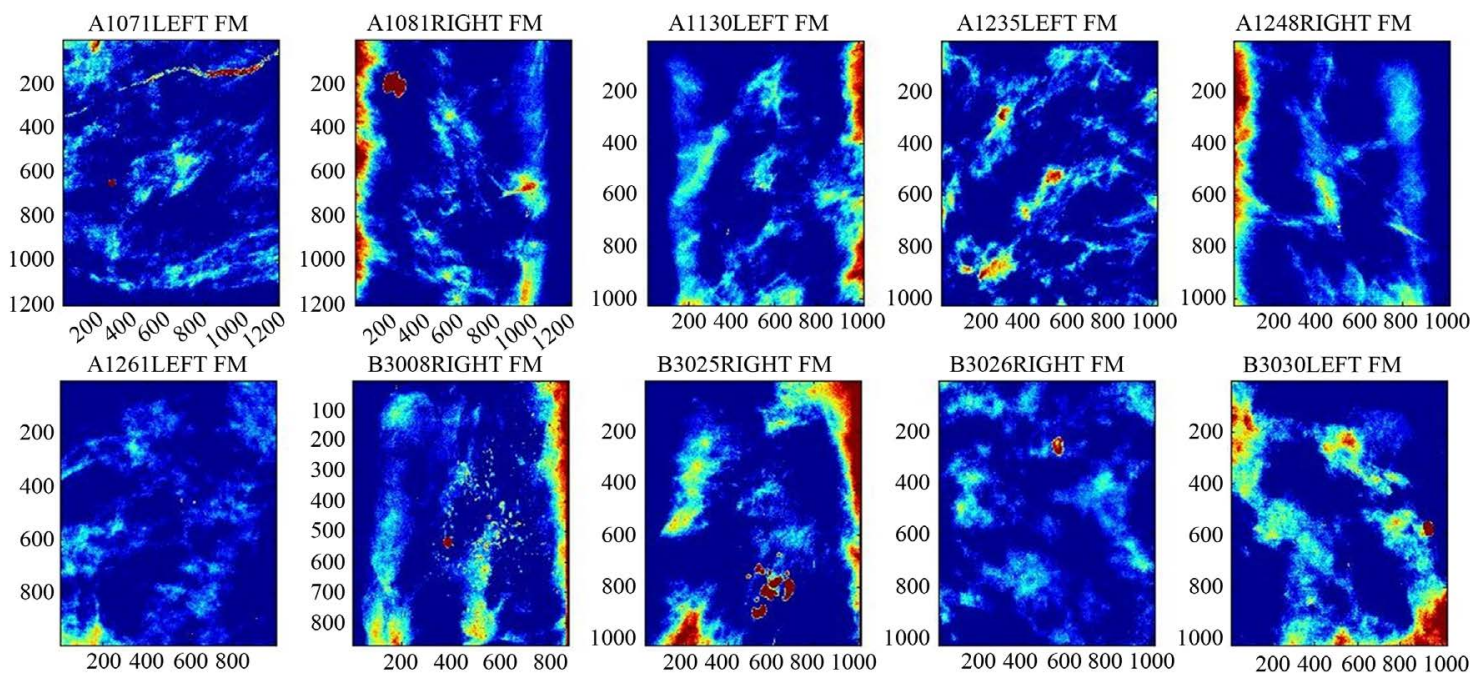

Figure 9. Frequency space filtering spectrogram with size $1024 \times 1024$. 

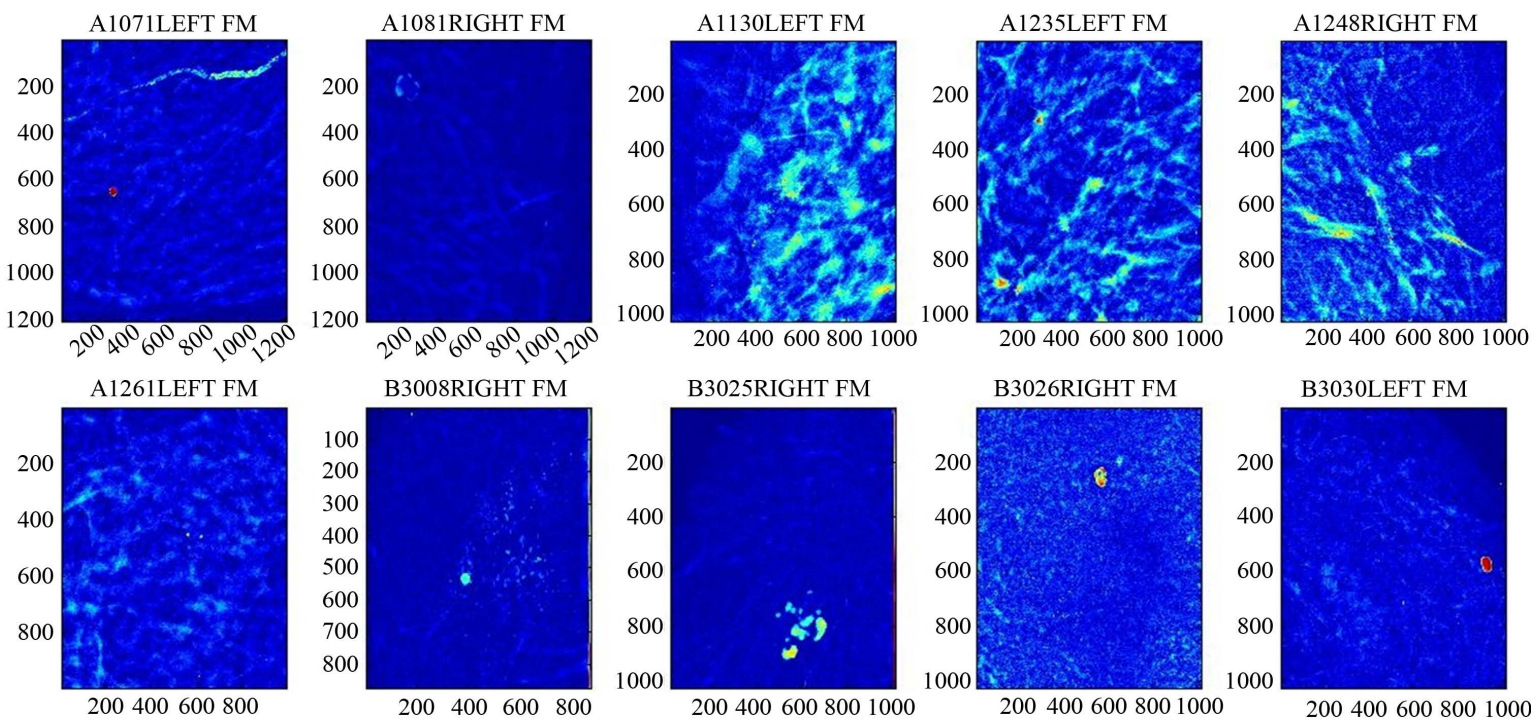

Figure 10. Morphologic filtering spectrograms with size $1024 \times 1024$ (With ball filtring).

Table 1. Time graphic of frequency space preprocessing algorithms.

\begin{tabular}{|c|c|c|c|c|c|c|c|c|c|}
\hline \multirow{2}{*}{ Images } & Ball & Diamond & Disk & Line & Octagon & Pair & Periodic Line & Rectangle & Square \\
\hline & Time (s) & Time (s) & Time (s) & Time (s) & Time (s) & Time (s) & Time (s) & Time (s) & Time (s) \\
\hline 1 & 5 & 1.3 & 2.7 & 1.5 & 2.7 & 1 & 2.3 & 2 & 1.7 \\
\hline 2 & 10 & 2.5 & 4.7 & 2.8 & 4.9 & 2.1 & 4.2 & 3.5 & 3.3 \\
\hline 3 & 14.7 & 4 & 7.3 & 4.4 & 7.5 & 3.4 & 6.4 & 5.3 & 5 \\
\hline 4 & 20 & 5.7 & 10.1 & 6.6 & 10.4 & 4.7 & 9 & 7.2 & 7 \\
\hline 5 & 25.2 & 7.4 & 12.9 & 7.9 & 13.1 & 6.2 & 11.5 & 9.2 & 9 \\
\hline 6 & 30.4 & 9.5 & 15.8 & 9.9 & 16.3 & 7.8 & 14.1 & 11.6 & 11.5 \\
\hline 7 & 36.1 & 11.4 & 19 & 12.1 & 19.3 & 9.7 & 17.5 & 13.7 & 13.8 \\
\hline 8 & 41.7 & 13.6 & 22.3 & 14.3 & 22.8 & 11.7 & 20.1 & 16.5 & 16.5 \\
\hline 9 & 47.4 & 16.7 & 25.7 & 17.1 & 26.9 & 14.3 & 23.5 & 19.1 & 19.4 \\
\hline 10 & 53.4 & 19 & 29.7 & 19.5 & 30.4 & 16.3 & 27.2 & 22.6 & 21.9 \\
\hline 20 & 129 & 57.5 & 79.7 & 61.3 & 77.6 & 49.9 & 72.6 & 63.6 & 65 \\
\hline 30 & 217.5 & 107.1 & 150 & 120.2 & 144.2 & 104.2 & 139.6 & 126.3 & 127 \\
\hline 40 & 335.4 & 179 & 215.3 & 178.4 & 237.7 & 183.4 & 234.9 & 209.1 & 210.1 \\
\hline 50 & 434.5 & 307.3 & 368.3 & 295.8 & 359.4 & 298.6 & 355.5 & 325.5 & 325.5 \\
\hline 60 & 556.3 & 361.3 & 491.1 & 446.6 & 507.9 & 426.9 & 504.4 & 461.9 & 463.9 \\
\hline
\end{tabular}

SP1 $\times$ 64. Table 1 and Table 2 represent the times for each of the filters used, each with their sub filtering in the frequency and space morphology.

Performance graphics were taken on every algorithm that was used through the investigation, show on Figure 11 and Figure 12. The x-axis contains information on number of images, while the y-axis contains information on time in seconds. 
R. O. Guardado-Medina et al.

Table 2. Time of morphologic space preprocessing algorithms.

\begin{tabular}{|c|c|c|c|c|c|c|}
\hline & $\begin{array}{l}\text { High Pass } \\
\text { Butterworth }\end{array}$ & $\begin{array}{l}\text { High Pass } \\
\text { Gaussiano }\end{array}$ & High Pass Ideal & $\begin{array}{c}\text { Low Pass } \\
\text { Butterworth }\end{array}$ & $\begin{array}{l}\text { Low Pass } \\
\text { Gaussiano }\end{array}$ & Low Pass Ideal \\
\hline & $\begin{array}{c}\text { Time } \\
\text { Processing (s) }\end{array}$ & $\begin{array}{c}\text { Time } \\
\text { Processing (s) }\end{array}$ & $\begin{array}{c}\text { Time } \\
\text { Processing (s) }\end{array}$ & $\begin{array}{c}\text { Time } \\
\text { Processing (s) }\end{array}$ & $\begin{array}{c}\text { Time } \\
\text { Processing (s) }\end{array}$ & $\begin{array}{c}\text { Time } \\
\text { Processing (s) }\end{array}$ \\
\hline 1 & 0.342 & 0.269 & 0.232 & 0.32 & 0.231 & 0.215 \\
\hline 2 & 0.68 & 0.436 & 0.525 & 0.664 & 0.527 & 0.495 \\
\hline 3 & 1.023 & 0.646 & 0.695 & 1.109 & 0.659 & 0.631 \\
\hline 4 & 1.864 & 0.891 & 0.887 & 1.358 & 0.901 & 0.834 \\
\hline 5 & 1.98 & 1.122 & 1.161 & 1.63 & 1.193 & 1.049 \\
\hline 6 & 2.476 & 1.391 & 1.348 & 1.904 & 1.38 & 1.249 \\
\hline 7 & 2.78 & 1.548 & 1.482 & 2.261 & 1.619 & 1.475 \\
\hline 8 & 3.161 & 1.76 & 1.79 & 2.639 & 1.783 & 1.725 \\
\hline 9 & 3.83 & 2.02 & 2.08 & 2.837 & 2.076 & 1.876 \\
\hline 10 & 4.103 & 2.225 & 2.213 & 3.137 & 2.294 & 2.068 \\
\hline 20 & 8.883 & 4.602 & 4.893 & 6.401 & 4.671 & 4.28 \\
\hline 30 & 12.332 & 7.209 & 7.712 & 9.799 & 6.973 & 6.664 \\
\hline 40 & 16.381 & 9.577 & 9.858 & 12.929 & 9.242 & 8.819 \\
\hline 50 & 20.851 & 12.151 & 12.642 & 16.121 & 11.379 & 11.526 \\
\hline 60 & 22.732 & 15.313 & 14.955 & 21.23 & 13.973 & 12.993 \\
\hline
\end{tabular}

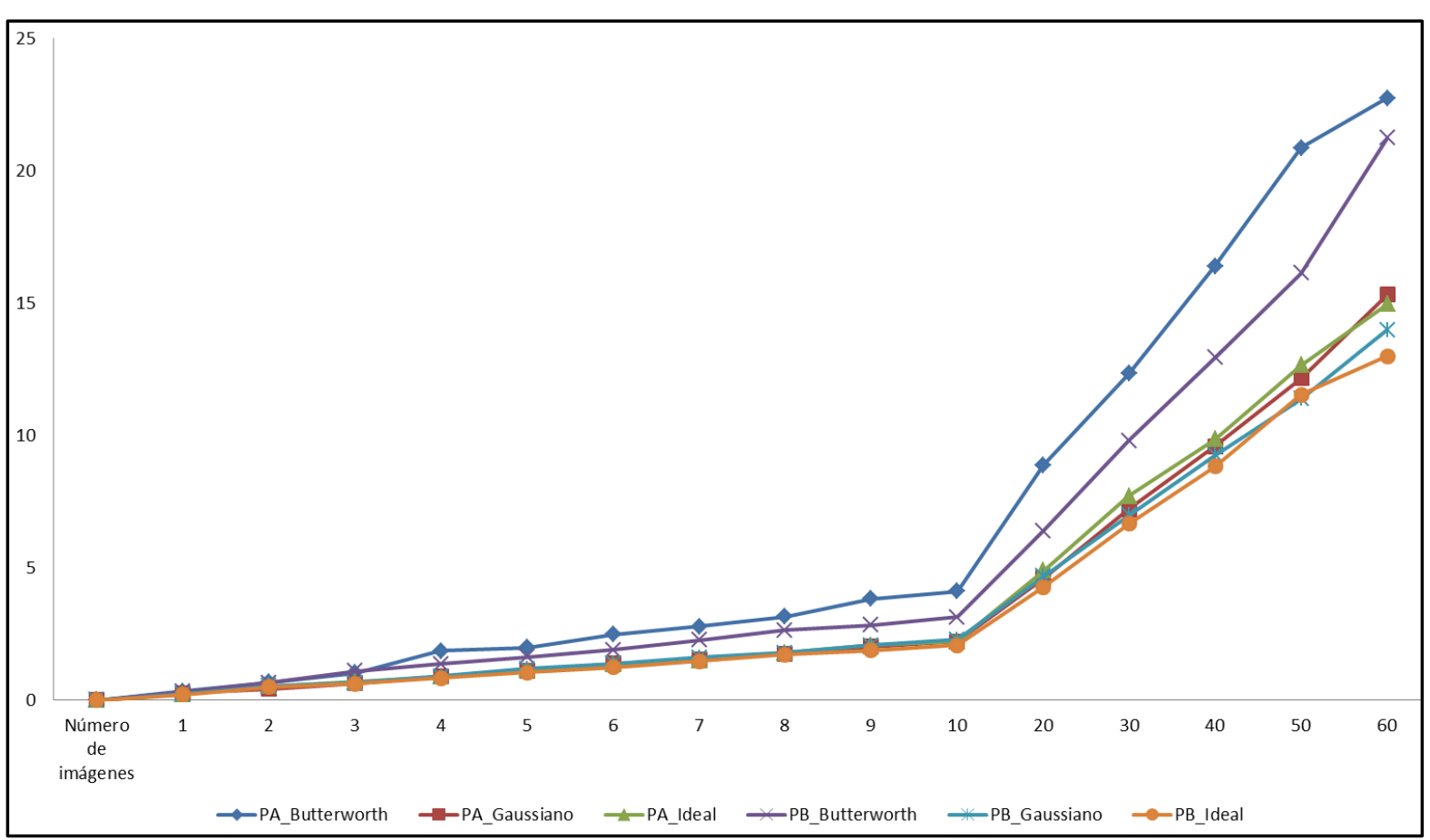

Figure 11. Time graphic of frequency space preprocessing algorithms. 


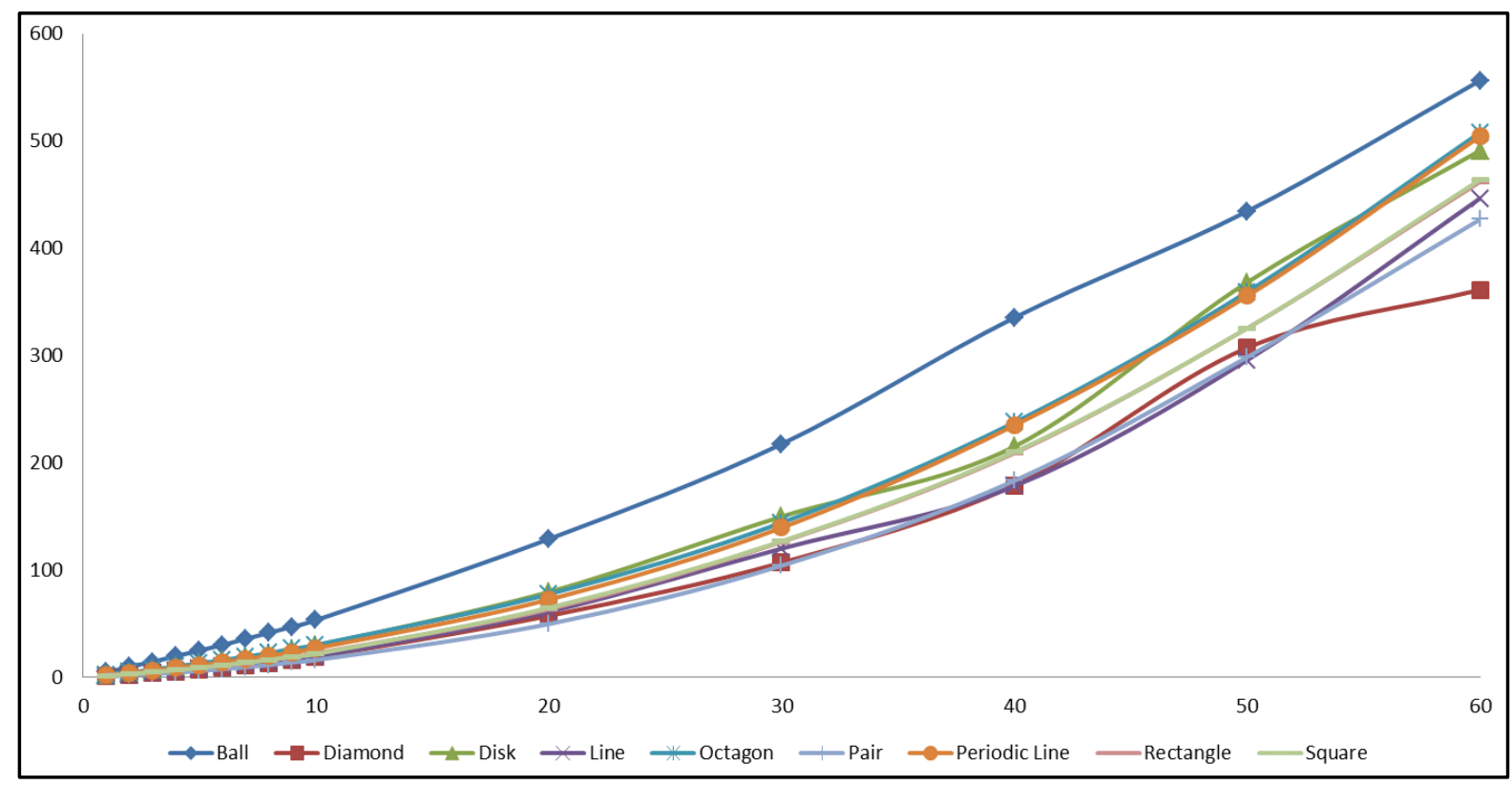

Figure 12. Time graphic of morphologic space preprocessing algorithms.

\section{Discussion}

Analyzing frequency space filtering, where its functionality is described, the higher the order, the closer it gets to its cutoff frequency. However, in real world testing, higher orders can be counterproductive for this investigation field, because there is a peak that overpasses cutoff frequency, allowing undesired frequencies to pass, the above makes that the filter shows some tissue as seen on Figure 6, and it can be checked on Figure 7, where accumulation of energy shows microcalcifications and tissue. This process is called resolution spectrogram window $1024 \times 1024$, which shows on similar colors the energy that represents microcalcifications and some energy accumulations on tissue, mixing up microcalcifications and tissue energies. Results can be seen on Figure 9. Regarding morphology filtering there is a noticeable separation between tissue and microcalcifications, where a more homogeneous filtering can be seen. Even if the size of the window is modified, by half the size for example, image quality is affected and visualization of microcalcifications is influenced. However, if the size of the window is increased, more memory is required on the processing, and the spectrogram can be seen on Figure 9 for morphology filtering. Finally, on every filter, lots of images were analyzed on time testing where the sample is 60 cases from 3 different volumes (cancer_01, cancer_04 and cancer_07), from 15 cancer volumes previously diagnosed (25 cases from cancer_01, 14 cases from cancer_04 and 21 cases from cancer_07). For frequency space filtering according to the graphic shown on Figure 10, times are low, meaning a fast processing, and every sub-filter is shown on the graphic and each sub-filter process from 1 image to 60 images, getting the lowest time: 13.973 seconds on Gaussian filter, and 22.732 seconds on high-pass Butterworth. Morphology filtering shows a slower processing: going from 363.1 to 556.3 seconds. The last point shows different times, although the frequency is faster than morphological space, and the morphological filters (Ball filtering) shows a significant result than frequency filters. That situation is supported on Figure 9 vs Figure 10. Finally, one of the benefits of image preprocessing is to enhance the characteristics of particular interest, which facilitates future processing steps (clustering, classification even neural networks).

\section{References}

[1] International Agency for Research Cancer (2013) http://www.iarc.fr/

[2] Instituto Nacional de Cancerología de México, Sistema de información de Cáncer (2013) http://www.infocancer.org.mx/contenidos.php?idcontenido=1

[3] Instituto Nacional de Estadística y Geografía (2014) http://www.inegi.org.mx/

[4] Instituto Nacional de Cáncer de los Institutos Nacionales de la Salud en EE.UU (2013) 
http://www.cancer.gov/espanol/recursos/hojas-informativas/deteccion-diagnostico/mamografias.

[5] Quintanilla-Dominguez, J., Cortina-Januchs, M.G., Ojeda-Magana, B., Jevti, A., Vega-Corona, A. and Andina, D. (2010) Microcalcification Detection Applying Artificial Neural Networks and Mathematical morphology in Digital Mammograms. World Automation Congress.

[6] Guardado-Medina, R.O, Ojeda-Magaña, B., Quintanilla-Domínguez, J., Ruelas, R. and Andina, D. (2013) Quality of Microcalcification Segmentation in Mammograms by Clustering Algorithms. SOCO13, Salamanca, Spain.

[7] Ojeda-Magaña, B., Quintanilla-Dominguez, J., Ruelas, R. and Andina, D. (2009) Images Subsegmentation with the PFCM Clustering Algorithm. 7th IEEE International Conference on Industrial Informatics, 23-26 June 2009, Cardiff, 499-505.

[8] Malar, E., Kandaswamy, A. and Gauthaam, M. (2013) Multiscale and Multilevel Wavelet Analysis of Mammogram Using Complex Neural Network. Springer International Publishing, Switzerland, 658-668.

[9] Chen, Z., Strange, H., Denton, E. and Zwiggelaar, R. (2014) Analysis of Mammographic Microcalcification Clusters Using Topological Features. Springer International Publishing, Switzerland, 620-627.

[10] Paradkar, S. and Spande, S. (2011) Intelligent Detection of Microcalcification from Digitized Mammograms. Sadhana, 36, 125-139.

[11] Gonzalez, R.C., Woods, R.E. and Eddins, S.L. (2004) Digital Images Processing Using Matlab. Pearson Education.

[12] Castleman, K.R. (1999) Digital Images Processing. Prentice Hall, Upper Saddle River.

[13] Gonzalez, R. C. and Woods, R.E. (1996) Tratamiento Digital de Imagines. Editorial Díaz de Santos, S.A.

[14] Rose, C., Turi, D., Williams, A., Wolstencroft, K. and Taylor, C., IWDM (2006) The Digital Database for Screening Mammography, University of South Florida. http://marathon.csee.usf.edu/Mammography/Database.html 